Meta

Journal des traducteurs

Translators' Journal

\title{
Les noms propres dans la traduction littéraire
}

\section{Hanne Martinet}

Volume 27, numéro 4, décembre 1982

URI : https://id.erudit.org/iderudit/003590ar

DOI : https://doi.org/10.7202/003590ar

Aller au sommaire du numéro

Éditeur(s)

Les Presses de l'Université de Montréal

ISSN

0026-0452 (imprimé)

1492-1421 (numérique)

Découvrir la revue

Citer cet article

Martinet, H. (1982). Les noms propres dans la traduction littéraire. Meta, 27(4),

392-400. https://doi.org/10.7202/003590ar d'utilisation que vous pouvez consulter en ligne.

https://apropos.erudit.org/fr/usagers/politique-dutilisation/ 


\title{
LES NOMS PROPRES DANS LA TRADUCTION LITTÉRAIRE*
}

\author{
HanNe Martinet
}

Les remarques qui suivent sont le résultat de nos réflexions à propos du transfert des noms propres d'une langue à une autre et ce, dans des textes écrits. Nos remarques sont basées sur la traduction d'un roman effectuée l'année dernière. Il s'agit du roman danois Creme fraiche de Suzanne Brøgger, en français Crème fraîche.

Le nombre et la diversité des noms propres y étaient tels que cela nous a menée, d'une part, à constater que donner des notes en bas de pages serait excessif, d'autre part, à nous demander si de telles notes sont toujours une procédure de transfert adéquate et enfin, sinon à reprendre les discussions antiques ou contemporaines à ce sujet, du moins à reprendre en considération les définitions traditionnelles des noms propres. Nombreux chercheurs (figurant groupés dans la bibliographie) considèrent en effet, avec Alain Rey, qu'ils « désignent des individus ou des réalités individuelles $\gg$ (le Petit Robert 2, Paris, 1974, p. XI).

La définition n'est pas inexacte. Cependant, lorsqu'on considère, d'une part, les noms propres dans la diversité des textes où ils sont utilisés et où, comme nous allons le voir, on ne peut pas dire qu'ils sont toujours de simples denotata, ou «étiquettes» comme disent certains, il nous semble que l'on pourrait élargir le raisonnement et dire qu'ils peuvent aussi être indices dans l'utilisation que fait Éric Buyssens de ce terme dans la Communication et l'articulation linguistique. Un nom propre comme Champs-Elysées peut indiquer l'avenue, la station de métro ou, dans un roman, être utilisé comme symbole de ce qu'est Paris pour certains. On peut aussi percevoir tout ce que peuvent susciter en nous aujourd'hui même, et dans le monde entier sans doute, des noms comme Afghanistan et Iran ou ce que pouvait susciter un nom comme Viêt-nam dans un passé encore récent.

D'autre part, bien que des noms comme Greta Garbo ou Manhattan puissent être utilisés dans la plupart des langues occidentales sans changement de graphie, ils pourront dans la langue-cible et dans certains textes nécessiter une explication, donnée soit en note soit dans le texte même. Pour ce qui est de noms comme København et Helsing $\phi r$, ils peuvent s'épeler différemment. Ces deux noms de villes s'écrivent Copenhague et Elseneur en français, par exemple

\footnotetext{
* Colloque de Glendon, 1980.
} 
et, de plus, Elseneur (et peut-être aussi Copenhague) pourra également, dans la langue-cible, nécessiter une explication.

Pour ce qui est enfin du nom d'une petite ville danoise comme Funder, ou d'un nom comme søerne (les lacs qui se trouvent dans Copenhague), ils réclament, dans un roman, soit une note soit une explication dans la langue-cible. Ceci vaut peut-être également, pour des noms comme H.C. Ørsted et J.A. Schade, un poète danois qui n'est sans doute connu que d'un nombre restreint de Français, ses traductions étant récentes.

Il va sans dire que ces noms - dans d'autres textes - peuvent être utilisés sans changement ni explication. On pourra penser ici aux brochures touristiques ou aux cartes géographiques par exemple, où il pourra être utile de donner d'abord la graphie locale du nom propre suivie de sa graphie dans la langue-cible et éventuellement d'une explication pour aider l'étranger à trouver son chemin.

Le transfert des noms propres ne pouvant donc pas se réduire à être une simple question de graphie - ce dont on devra par exemple tenir compte dans les banques de données - il nous a semblé qu'il pourrait être adéquat de catégoriser les noms propres d'un roman pour essayer de trouver sinon des règles du moins des principes de transfert.

Le roman qui nous intéresse ici est écrit à la $1^{\text {re }}$ personne du singulier. On y suit l'évolution d'un je-narrateur exubérant, l'auteur, qui raconte son enfance au Danemark, son adolescence en Asie du Sud-Est, ses voyages de par le monde, ses multiples rencontres, ses idées sur la société d'aujourd'hui, sur la littérature, l'art, etc., et ses phantasmes. Ce faisant, elle utilise une multitude de noms propres - dont certains manifestent des faits culturels, géographiques, historiques, etc., en bref tout ce que nous appellerons ici culture dans l'acception la plus large du mot - et dont la dynamique est en perpétuel mouvement.

Nous allons d'abord expliquer très brièvement l'esquisse de catégorisation d'un tout petit nombre des noms propres du roman donnée p. 19 et en examiner ensuite les fonctions.

Nous nous plaçons d'abord dans l'optique de ce qu'il est convenu d'appeler récepteur et qui est ici lecteur. Pour faire cette catégorisation, nous nous sommes basée sur la forme des noms propres et sur nos connaissances du monde mais, surtout, sur l'utilisation qui en est faite à l'intérieur de l'univers qu'est le roman. Nous distinguons également entre association et connotation. Le premier concept désigne les représentations que peut évoquer un nom propre chez une personne. Le second concept désigne les représentations que peut évoquer un nom propre chez un groupe de personnes ou une communauté. Nous inspirant de ce que dit Claude Germain dans la Notion de situation en linguistique, nous distinguons également entre contexte linguistique immédiat, c'està-dire les éléments linguistiques réalisés juste avant ou après un élément $X$, et contexte linguistique non immédiat, c'est-à-dire tout le texte.

À l'intérieur de cet univers qui est créé dans le roman, nous avons distingué deux champs : un champ non fictionnel et un champ fictionnel. 
Le champ non fictionnel est l'époque, et donc la culture dans lesquels l'auteur a situé son je-narrateur - époque et culture qu'elle suppose connues du lecteur danois.

Pour transmettre cette culture, l'auteur a entre autres choisi ce que nous avons appelé les indices de culture d'une part, et les indices d'imaginaire, d'autre part.

Les indices de culture sont divisés en deux catégories : $(\mathrm{X})$ ceux que l'auteur n'explicite pas par un contexte linguistique immédiat, sans doute parce qu'elle les suppose connus du lecteur danois et, $(Y)$ ceux qu'elle explicite souvent par un contexte linguistique immédiat comme «le docteur $X$ », «les tableaux mythologiques de $X_{»}$, sans doute parce qu'elle les suppose moins connus.

Certains ne sont cependant pas explicités, Buzhkhazi par exemple, mais à notre demande d'explicitation, l'auteur a répondu qu'elle était d'avis «qu'il fallait laisser une part de mystère dans un roman» - réponse qui n'est pas sans laisser perplexe un traducteur. Nous pouvons à ce sujet mentionner que Günther Grass tient des colloques avec ses traducteurs pour y discuter entre autres les problèmes d'interprétations.

Ces deux catégories sont ensuite chacune divisées en deux sous-catégories selon qu'on a affaire à des indices de culture danoise $\left(X_{1}\right)$ et $\left(X_{2}\right)$ ou étrangères $\left(\mathrm{Y}_{1}\right)$ et $\left(\mathrm{Y}_{2}\right)$.

Chacune de ces quatre sous-catégories peut ensuite être divisée en de multiples groupes : toponymes, anthroponymes, phénomènes culturels, etc.

Imbriqué dans ce champ non fictionnel, nous avons le champ fictionnel, l'intrigue du roman, qui, même s'il n'est peut-être pas toujours fictionnel pour l'auteur, n'en reste pas moins fictionnel pour le lecteur, entre autres, parce que certains noms propres n'étant que des prénoms, ils ne peuvent pas, de prime abord, fonctionner, dirons-nous, sur le même plan que les autres dans l'univers du roman. L'auteur y présente ce qui est personnel au je-narrateur, sa famille, ses amis, ses phantasmes, etc., c'est-à-dire tout ce qu'elle suppose inconnu du lecteur. Les noms propres utilisés sont sans doute des créations et par certains aspects, le roman est un roman-clé. Ce sont ces noms que nous avons appelés indices d'imaginaire.

Les indices d'imaginaire peuvent ici se diviser en deux catégories: (L) les noms de personnages, qui sont, comme nous l'avons dit, souvent des prénoms et, (M) les toponymes mythiques.

Pour la catégorie (L), il est intéressant de constater que, souvent, l'auteur n' 'explicite» pas à proprement parler les noms comme nous l'avons indiqué plus haut, mais qu'elle «présente» ces personnages par le biais de dialogues, de descriptions, de comportements, d'attitudes, etc. Ce contexte ne figure pas toujours immédiatement avant ou après le nom propre, et il peut n'être donné que bien plus tard : $M a x$, le héros masculin du roman, figure à la première page du livre pour n'être «présenté » qu'à la page 98.

Pour la catégorie (M), on n'a trouvé qu'un seul nom propre, Tiranstraedet ( $m$.àm. «le détroit de Tiran»). L'auteur l'ayant situé près de Gibraltar, il est clair qu'il ne s'agit pas du détroit du golfe d'Aqaba. 
Après cette brève explication de la catégorisation des noms propres, nous allons examiner les fonctions qu'ils peuvent avoir dans l'univers qu'est un roman.

Pour nous, lecteur, ce qui nous permet, par exemple de savoir que le déroulement des événements a lieu aujourd'hui, c'est, comme l'on s'en doute, les noms propres du champ non fictionnel comme Kate Millett, Gagarin et Godot. Ce qui nous permet de connaître les lieux ce sont des toponymes comme København, søerne, Virum, Bangkok et Värnamo, une ville suédoise.

Mais, en même temps que l'auteur nous communique l'époque et les lieux où se déroule l'intrigue par le biais des noms propres, elle transmet aussi par le contexte immédiat ou non immédiat - ses opinions ou celles d'autrui sur les phénomènes et personnalités culturels ainsi que ses impressions des lieux et des ambiances qu'elle y a trouvés. Elle peut ainsi parvenir à imprégner le lecteur de sa vision des choses, à l'imprégner de sa vision des entités qu'elle désigne par les noms propres ou, dit autrement, elle parvient à tisser des réseaux d'associations autour de noms propres inconnus. Elle parvient aussi à défaire des faisceaux de connotations qui sont liés à des entités connues et ainsi, à tisser de nouveaux réseaux d'associations autour de celles-ci. Elle parvient comme le dit Victor Hugo à «faire dans l'esprit des autres une petite incision où l'on met une idée à soi ». (L'homme qui rit, II ${ }^{\mathrm{e}}$ partie, livre $\mathrm{V}$, chap. 2 ). Ses descriptions de Viêt-nam, de New York et autres, en sont des exemples.

Pour ce qui est maintenant des noms propres des catégories $\left(X_{1}\right)$ et $\left(X_{2}\right)$, ils sont le plus souvent réalisés sans contexte immédiat - précisément parce que l'auteur suppose - à tort peut-être parfois - que le lecteur danois non seulement les connaît, mais connaît aussi les faisceaux de connotations qui peuvent y être rattachés dans la société danoise. Cela ne veut pas dire qu'elle ne parvienne pas, là aussi par le biais du contexte, à faire de petites incisions dans 1 'esprit du lecteur.

Nous pouvons ainsi constater que les noms propres du champ non fictionnel de ce roman, outre qu'ils fonctionnent comme denotata, peuvent aussi fonctionner comme indices de culture et transmettre au lecteur ce qu'André Martinet appelle «vision des choses» dans «Poésie, connotations et culture», et dont il dit que «les termes qui désignent ces choses impliquent une masse d'ineffable que le poète saura transmettre à autrui en jouant sur la langue " (ibid., nous soulignons). Ils participent ainsi, avec les autres éléments linguistiques ou monèmes, à donner au lecteur la possibilité d'interpréter le roman et de mieux le "saisir», comme le dit Georges Mounin dans La littérature et ses technocraties.

Nous allons maintenant examiner les fonctions des noms propres du champ fictionnel.

Ce qui nous permet de supposer qu'il s'agit de fiction, c'est d'abord qu'on a surtout des prénoms comme Søren, Jean-Louis ou Max qui, de prime abord, ne suscitent aucune association qui puisse fonctionner comme nous l'avons dit dans l'univers du roman, si ce n'est qu'ils portent une indication ethnique. $\mathrm{Ce}$ n'est que petit à petit que le lecteur peut se faire une idée de ces personnages. Au fil des années, par l'échange avec autrui, ces associations pourront finir par se fixer en faisceaux de connotations qui pourront devenir collectives 
ou quasi collectives et, avec les ans, pseudonymes et toponymes mythiques pourront devenir indices de culture. C'est ce qu'on avait pour Zeus, Godot, etc.

Ainsi, outre qu'ils fonctionnent comme denotata, ces noms propres fonctionnent primordialement comme indices ethniques pour les personnages du roman. Ces indications sont données à l'aide de leurs diverses graphies, qu'il s'agisse de noms courants $\left(L_{1}\right)$ ou de sobriquets $\left(L_{2}\right)$. Cette utilisation suppose évidemment que le lecteur soit relativement familier avec ces graphies et sonorités. Dans nos sociétés voyageuses et imprégnées de mass-media, cela ne devrait pas poser au lecteur trop de problèmes.

Pour les Danois, les noms de la catégorie $\left(\mathrm{L}_{1}\right)$ comme Søren, Bodille et $B \emptyset r g e$ ont des graphies et des sonorités danoises, le dernier ayant actuellement des connotations dépréciatives et ridicules, des noms comme Jean-Louis, des graphies et des sonorités françaises. Max, par contre, qui est un diplomate dans le roman, peut être considéré comme un indice d'appartenance à un milieu plus international.

Dans le groupe $\left(\mathrm{L}_{2}\right)$, on retrouve les mêmes indices nationaux dans les sobriquets. Danois sont des noms comme Jørgen Syltetøj (= Jørgen Confiture). Non danois sont des noms comme Alfoncus et John Forever.

Pour ce qui est enfin du seul toponyme mythique, on a une graphie danoise dans -straedet (= détroit) et non danoise dans Tiran.

Avant de terminer, nous avons choisi de montrer à l'aide d'un exemple le transfert du toponyme Virum - qu'une note explicative ne représente peutêtre pas toujours une procédure de transfert adéquate, d'une part et, d'autre part, que les définitions traditionnelles des noms propres devraient être élargies.

Dans l'énoncé suivant «Personnellement, je préfère de beaucoup la misère visible, comme ça je peux la voir, parce qu'elle existe, même cachée derrière les pavillons en briques jaunes et les plantes vertes des bien-pensants danois » (Crème fraîche, p. 26), ce sont les éléments «bien-pensants danois» qui représentent le transfert de «Virum», qui est une banlieue de Copenhague où habitent aujourd'hui des gens appartenant surtout à la classe moyenne et dirigeante danoise. Le transférer directement en derrière les «plantes vertes à Virum», ce qui aurait correspondu exactement à l'énoncé en danois, avec une note donnant cette explication, ne nous semblait cependant pas pouvoir susciter l'association qu'avait voulu éveiller l'auteur dans cet alinéa, où elle critique certains aspects de la culture danoise, et ceci pour deux raisons.

Sur un plan plus pratique d'abord - mais auquel nous sommes d'avis qu'il faut attacher une certaine importance - il peut être gênant, voire agaçant, lorsqu'on lit un roman d'être contraint à interrompre le cours de la lecture et donc le cours du processus associatif - pour lire les spécifications données en notes. C'est du moins ce que plusieurs personnes nous ont dit. Et, si le nombre des notes que l'on doit donner est élevé, il est peut-être préférable d'insérer ces «explications» dans le texte même, comme le fait d'ailleurs l'auteur lui-même pour les noms propres de la catégorie (Y) qu'elle suppose moins connus du lecteur danois. C'est la procédure que nous avons suivie ici.

Ensuite, sur le plan associatif, une note qui expliquerait ce qui a été dit plus haut, serait erronée car en utilisant Virum ici, l'auteur ne voulait 
désigner ni cette banlieue ni ses habitants mais les Danois qui se conforment à des valeurs sociales et morales conservatrices et à l'ordre établi des bienpensants. Dans une éventuelle note donnée pour ce Virum, il aurait donc fallu expliquer le denotatum puis le contenu de l'indice de culture qu'il est aussi, ce qui aurait été extrêmement long et peut-être aussi difficile à saisir.

Or comme il ne fonctionne ici que comme indice de culture - c'est l'équivalence stylistiquement adaptée au contexte immédiat qui nous a paru pouvoir être le mieux à même de susciter chez le lecteur français une association analogue à celle que pouvait avoir eue le lecteur danois et que nous avions eue nous-même.

Il est sans doute utile de souligner aussi que Virum n'était pas porteur de connotations spécifiques en 1978 lorsque l'auteur l'a utilisé comme indice de culture, mais qu'il le deviendra peut-être puisque Suzanne Brøgger est un auteur connu au Danemark et que nous en parlons.

On pourrait tirer bon nombre de conclusions de cette esquisse. Nous nous en tiendrons cependant à celles qui sont pertinentes pour le traducteur, le lexicologue et l'informaticien, et ici, pour une traduction d'une langue occidentale à une autre langue occidentale.

Il devrait ressortir de ce qui précède que dans aucun des champs distingués l'utilisation des noms propres n'est pas arbitraire dans tous les cas, supposition que l'auteur nous a confirmée. L'interprétation du roman est donc fonction de ces éléments.

Si l'on part alors de ce qu'Eugène Nida appelle principe d'équivalence fonctionnelle dans Language Structure and Translation pour dire qu'un ouvrage littéraire traduit doit produire le même effet sur le lecteur étranger que sur le lecteur de l'original, et lui donner la même «vision des choses», il s'agit pour ce qui est des noms propres, entre autres, de choisir des éléments linguistiques qui peuvent susciter chez le lecteur étranger des associations analogues à celles que peut avoir le lecteur danois et, pour emprunter le terme qu'utilise Claude Tatilon dans Sonorités et textes poétiques, dont les «impressivités » sont identiques dans les deux langues.

Un des principes directeurs était ici de transférer les aspects non fictionnel et fictionnel du roman tout en conservant les aspects «cosmopolites» de ce dernier.

Nous ne pouvons pas entrer ici dans tous les détails des problèmes que posent les transferts d'impressivité, ni sur les pertes inévitables. Les connotations dépréciatives et ridiculisantes qu'a actuellement par exemple le prénom Børge au Danemark sont impossibles à transférer lorsqu'on doit garder ce prénom comme pseudonyme ethnique. La poésie et la luminosité que peut évoquer sqerne (dans $\mathrm{X}_{1}$ ), dans une certaine mesure aussi. Mais on pourra essayer de procéder par compensation comme le soulignent Jean-Paul Vinay et Jean Darbelnet dans Stylistique comparée du français et de l'anglais. Pour ce qui est des aspects-clé du roman, ils sont en partie perdus pour le lecteur français lorsqu'il s'agit de pseudonymes danois.

Certains des noms propres des catégories (Y), (L) et (M) étant, eux, spécifiés par un contexte immédiat ou non immédiat, leur transfert est sans doute 
un peu plus facile que pour ceux de la catégorie $\left(\mathrm{X}_{1}\right)$, comme nous venons de le voir. Pour ceux de la catégorie $\left(X_{2}\right)$, il s'agissait de déterminer le degré de notoriété des noms en fonction du public français qui, pour un roman, n'est pas toujours facile à cerner.

Par tout ce qui précède, nous espérons être parvenue à montrer que les noms propres ne peuvent pas être traités uniquement comme denotata et qu'on pourrait alors élargir la définition donnée plus haut pour dire avec Oswald Ducrot et Tzvetan Todorov par exemple, qu'«on peut alors considérer comme le sens d'un nom propre pour une collectivité donnée un ensemble de connaissances relatives au porteur de ce nom $\gg$ (Dictionnaire encyclopédique des sciences du langage).

Ce que cette définition ne fait pas ressortir assez nettement cependant, c'est ce qu'on pourrait appeler la «dynamique des noms propres» - c'est-àdire que de denotata, ils peuvent passer au stade d'indice de culture et vice versa, et que du stade d'indices d'imaginaire, ils peuvent passer au stade d'indices de culture.

C'est aussi sans doute ce qu'entendait Otto Jespersen lorsqu'il écrivait «The first time you hear of a person or read his name in a newspaper, he is «a mere name» to you, but the more you hear and see of him the more will the name mean to you» (The Philosophy of Grammar).

Pour le traducteur, il s'agira alors d'abord de savoir si un nom propre donné dans un texte donné est denotatum, indice de culture ou les deux à la fois. De savoir ensuite s'il est porteur d'un faisceau de connotations spécifiques ou d'un réseau d'associations propres à l'utilisateur. Et enfin, dans la languecible, de choisir des éléments linguistiques qui sont aptes à susciter des associations analogues à celles que peut avoir eues le lecteur de l'ouvrage original.

Ce qui resterait alors à faire ce serait d'essayer de déterminer les faisceaux de connotations qui sont liés aux noms propres dans une société donnée.

Avant de terminer, nous tenons à reprendre brièvement un point que nous n'avons pas pu traiter ici et qui sera approfondi ailleurs. Nous disions plus haut que les noms propres de l'ouvrage participaient à donner au lecteur la possibilité d'interpréter le roman. À ce verbe, nous opposerons le verbe comprendre. Vingt-cinq ans d'activité traduisante de textes extrêmement diversifiés nous ont en effet enseigné, entre autres, qu'il est nécessaire de toujours envisager un texte dans sa globalité et, ainsi, d'envisager les éléments linguistiques que l'on va traduire à l'intérieur de cette globalité - de cette somme d'indices qu'est un texte.

Nous expliquerons comprendre d'abord. Certains textes sont rédigés dans le but de communiquer ce qu'on pourrait appeler une «information brute». Nous pensons ici aux tarifs douaniers, modes d'emploi, à certaines recettes de cuisine sans «ornement linguistique», etc. Ils exigent du lecteur des connaissances précises. On peut ne pas comprendre ces textes, soit parce qu'on n'a pas les connaissances nécessaires soit parce qu'ils sont mal rédigés - mais il pourra être dangereux ou malencontreux d'interpréter les incorrections ou les ambiguités rencontrées. Et donc de les traduire sans avoir eu recours à des spécialistes dans les divers domaines en cause. 
D'autres textes transmettent au lecteur ce qu'André Martinet appelle "visions des choses", comme nous venons d'en voir un exemple. Nous mettons clans cette catégorie tous les textes qui ne consistent pas uniquement en «information brute». Outre qu'ils exigent certaines connaissances précises de la part du lecteur, ces textes font appel à son sens psychologique, politique, esthétique, etc., à ses facultés de perception et à sa sensibilité. On peut très bien, là, «comprendre » les textes sans cependant pouvoir les interpréter ou, du moins, sans pouvoir leur assigner une signification unique - ceci valant également pour certains de leurs éléments linguistiques. Nous avons vu comment Günther Grass essaie de résoudre ces problèmes d'interprétation. Nous avons, nous, demandé à l'auteur de lire la traduction française du roman. Et il ne peut pas faire de doute que les traducteurs de traités internationaux par exemple devraient pouvoir avoir recours à leurs rédacteurs, car, comme le dit, M.A.K. Halliday dans Learning how to mean, Explorations in the Development of Language. "The semiotic structure of the environment - the ongoing social activity, the roles and statuses, and the interactional channels - both determines the meanings exchanged and is created by and formed out of them " (p. 143).

\section{BIBLIOGRAPHIE} denotata

Nous groupons d'abord certains ouvrages où l'on considère que les noms propres sont des

CHOMSKY, N., (1965): Aspects of the Theory of Syntax, The M.I.T. Press, Cambridge, Mass., p. 29 et 201.

GARDINER, A., (1940): The Theory of Proper Names, Oxford University Press, Londres. Dans cet ouvrage l'auteur discute les points de vue de l'Antiquité et de J.S. Mill sur les noms propres.

GARDINER, A. (1963): The Theory of Speech and Language. Clarendon Press, Oxford.

LYONS, J. (1971): Introduction to Theoretical Linguistics, Cambridge University Press, Londres, p. 337.

LYONS, J. (1977) : Semantics, Cambridge University Press, Londres, vol. I p. 224-225, vol. II, p. 644-646.

REY, A., le Petit Robert 2, S.E.P.R.E.T., Paris.

SEARLE, J.R. (1971) : "The Problem of Proper Names", dans Semantics, ed. STEINBERG, D.D., JAKOBOVITS, L. A., Cambridge University Press, Cambridge, p. 134-141.

VENDRYES, J. (1923, 1968) : le Langage, Albin Michel, Paris, p. 212.

Ouvrages cités dans la communication :

BRØGGER, S. (1978) : Creme fraiche, Rhodos, Copenhague.

BRÖGGER, S. (1979) : Crème fraîche, Belfond, Paris, traduit du danois par MARTINET, H.

BUYSSENS, E. (1970) : la Communication et l'articulation linguistique, Presses Universitaires de Bruxelles, Bruxelles.

DUCROT, $O$. et T. TODOROV, (1972) : Dictionnaire encyclopédique des sciences du langage, Seuil, Paris.

GERMAIN, C. (1973): la Notion de situation en linguistique, Éditions de l'Université d'Ottawa, Ottawa.

HALLIDAY, M.A.K. (1975) : Learning how to mean, Explorations in the Development of Language, Arnold, Londres.

JESPERSEN, O. (1924): The Philosophy of Grammar, Allen \& Unwin, Ltd., Londres.

MARTINET, A., «Connotations, poésie et culture», dans To Honor Roman Jakobson, vol. II, La Haye, Mouton, p. 1288-94.

MOUNIN, G. (1978): la Littérature et ses technocraties, Casterman, Paris.

NIDA, E.A. (1975): Language Structure and Translation, Stanford University Press, Stanford, Calif.

TATILON, C. (1976) : Sonorités et textes poétiques, Didier Canada, Ottawa.

VINAY, J.-P. et J. DARBELNET (1964): Stylistique comparée du français et de l'anglais, Didier, Paris. 


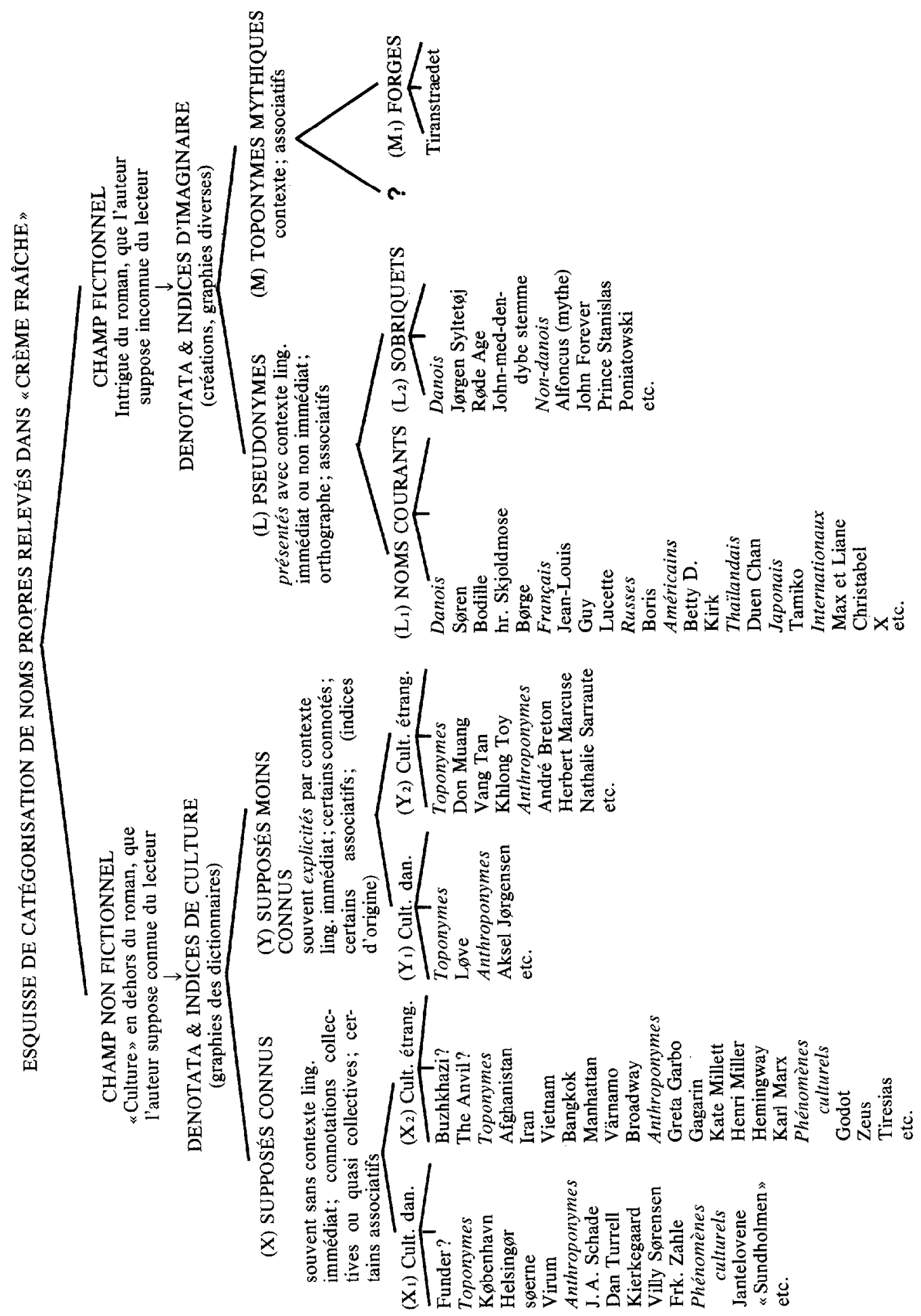

